\title{
Preparation of Stable Silver Nanoparticle Containing Glycerol-AOT [bis(2-ethylhexyl)sulfosuccinate sodium salt] Reverse Micellar Solution using Hydrogen Gas as Reducing Agent and Silver Nitrate as Precursor Material
}

\author{
Palash Setua \\ Dept. Of Chemistry, Pingla Thana Mahavidyalaya, Vidyasagar University, Midnapore, West Bengal, India. \\ *Corresponding Author: palashsetua @gmail.com, Tel.:+ 919474065807
}

Available online at: www.isroset.org

Received: 27/Sept/2019, Accepted: 15/Oct/2019, Online: 31/Oct/2019

\begin{abstract}
The research paper describes the synthesis of silver nanoparticles in nonaqueous AOT [bis (2-ethylhexyl) sulfosuccinate sodium salt] reverse micellar solution. Complete description of the reverse micellar system is glycerol-AOT-nheptane reverse micelle where AOT molecules act as surfactant molecule, glycerol as non aqueous polar core solvent and nheptane as nonpolar hydrocarbon which acts as the dispersion medium. In this study I have used glycerol as nonaqueous polar solvent which was used to create the AOT surfactant core of the reverse micelle and then incorporated silver ion $\left(\mathrm{Ag}^{+}\right)$using silver nitrate $\left(\mathrm{AgNO}_{3}\right)$ salt as precursor material responsible for metal nanoparticle formation. Freshly prepared hydrogen gas $\left(\mathrm{H}_{2}\right)$ is used as reducing agent to reduce silver ion $\left(\mathrm{Ag}^{+}\right)$to silver zero $\left(\mathrm{Ag}^{0}\right)$ state. Formation and precipitation of large particle was restricted by the cage like templating structure of reverse micelle. I have selected hydrogen gas as reducing agent because of its mild nature and also wanted to minimize the perturbation of natural reverse micelle environment.
\end{abstract}

Keywords-Metal Nanoparticle, Silver, Reverse Micelle, Nonaqueous solvent medium

\section{INTRODUCTION}

Conventional synthesis of metal nanoparticle and regulated morphological design has its long history [1-3]. Its application was tested in the field of electronics and photonics [3-4]. It was also used in catalyzing chemical process [5]. Researchers have shown that it can be used in the area of medicinal application to achieve the drug delivery process [6-7] and in various area of research [8$9,14]$. In the previous decade, all these work reported their complicated process and success in limited and controlled laboratory environment with complicated instrument setup. But if we consider the applicability of nanoparticle in real day to day life, then still it is very limited and rare. The reason is lack of simple and effective method of design which can be done with the simple laboratory setup available in school and colleges. This effort will create an atmosphere where 'nanoparticles' can be included in the school/college course curriculum, common student won't fear it as very complicated and sophisticated area limited only for research. Then it will be possible for them to systhesize the nanoparticle in their working laboratory environment and test their material efficacy in various known field. So in this paper I have described a very simple method for school/ college level chemistry students that can be used to synthesized silver nanoparticle directly using simple equipments like test tube, beaker, hydrogen gas and simple chemical substance like silver nitrate, AOT-surfactant (very similar to soap) molecule etc. Lastly this method is standardised in non-aqueous solvent medium so that nanoparticle containing solvent system can be directly used for simple chemical reaction as most of the organic reactions are done in non aqueous solvent medium.

Basic idea of this method is that we dissolve surfactant molecule in nonaqueous solvent medium like n-heptane above a certain concentration limit known as critical micelle concentration (CMC). Surfactant molecule AOT is basically an amphiphilic molecule, its one end is polar (known as head group) and the other end nonpolar (known as hydrocarbon tail). In this environment dissolved surfactant molecules form very small nanometre size globular aggregates known as reverse micelle. This reverse micellar structure has two main parts, one is polar core which is formed by the association of polar head group of a large number of surfactant molecule and the second is surface or interface part where all the nonpolar hydrocarbon tail parts reside. The structure of AOT reverse micelle is very much similar to the schematic shown in figure 1. In this study I have used the polar core of the reverse micelle and incorporated the 
silver ion using silver nitrate solution in nonaqueous polar solvent glycerol and reduced the silver ion to silver zero state using freshly prepared hydrogen gas as reducing agent. Size growth and agglomeration of silver atom is restricted by the cage like templating structure of the AOT reverse micelle.

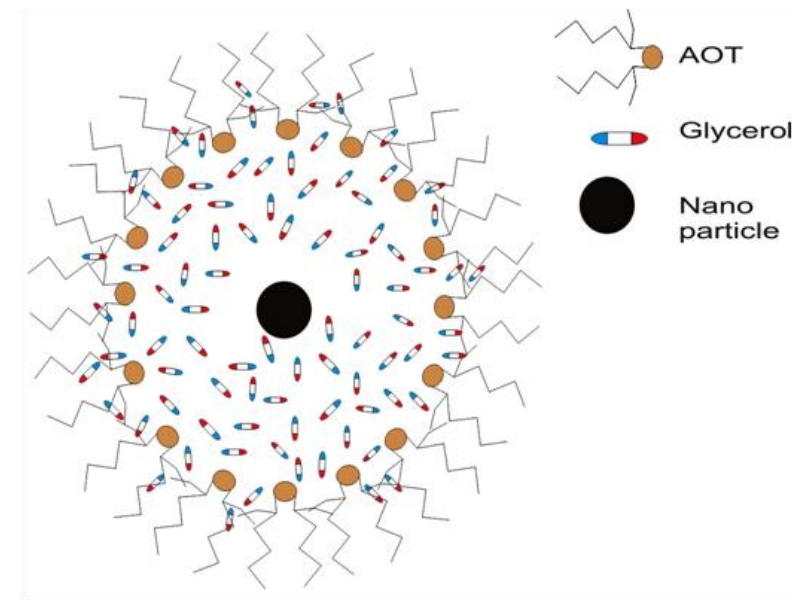

Figure 1. Schematic structure of AOT reverse micelle.

\section{RELATED WORK}

Use of reverse micelle as template medium for nanoparticle synthesis was developed and extensively studied by Pileni et al. [10]. But their working system and reverse micellar medium was developed in aqueous core environment. Later the field was extended to nonaqueous reverse micellar system by Sarkar et al.[11]. They have also successfully applied the technique in room temperature ionic liquid medium [12]. Now in this article, I am reporting the successful silver nanoparticle synthesis in nonaqueosu glycerol-AOT-n-heptane reverse micelle media.

\section{METHODOLOGY}

Materials: AOT [bis (2-ethylhexyl) sulfosuccinate sodium salt] was purchased from sigma and purified using standard process[13]. Silver nitrate was purchased from Merck and was used as received. Solvents glycerol and n-heptane were purchased from Merck and used as received.

Preparation of hydrogen gas: I used standard process and used the common reaction between iron sulphide and sulphuric acid to prepare hydrogen gas. Moisture present in the prepared gas was removed by passing it through a moisture absorbing column.

Instruments: TEM measurement was performed using a JEOL 2010 transmission electron microscope operating at $200 \mathrm{keV}$ with LaB6 filament. TEM samples was prepared by direct drop casting of nanoparticle contain reverse micellar solution on carbon coated $\mathrm{Cu}$ grid (300 mesh, Electron Microscopy Sciece). Dynamic Light Scattering (DLS) measurements were done using Nano ZS Malvern instrument employing a $4 \mathrm{~mW} \mathrm{He}-\mathrm{Ne}$ laser at wavelength $632.8 \mathrm{~nm}$. UV-Vis measurements were done using Shimadzu spectrophotometer.

Preparation of Reverse Micellar Solution using AOT in n-heptane nonpolar media: Concentration of AOT surfactant used for all reverse micelle solution was $0.1 \mathrm{M}$. Each reverse micellar solution is designated by a particular $\mathrm{w}$ value. It is nothing but the molar ratio of polar core solvent to surfactant. Simply,

$$
\mathrm{w}=\frac{\text { molar concentration of glycerol }}{\text { molar concentration of AOT }} \text {. }
$$

I used $\mathrm{w}=3$ condition for the present study. For reverse micellar solution preparation initially a stock solution of silver nitrate in glycerol is prepared. Its strength was $1.1 \mathrm{M}$. Then using this stock I prepared the reverse micellar solution where concentration of silver ion was $3 \times 10^{-3} \mathrm{M}$. Then freshly prepared hydrogen gas was bubbled through this solution for $2.5 \mathrm{hrs}$ at $298 \mathrm{~K}$. Initially the reverse micellar solution was colourless and transparent but after passage of hydrogen gas the solution become yellow in colour. This colour change was a direct indication of silver nanoparticle formation.

\section{RESULTS AND DISCUSSION}

Initially the stability of the pure glycerol-AOT-n-heptane reverse micellar solution was checked by ageing method. The results showed that the stability of the reverse micelle solution is very high and it remained stable for few weeks. Then I carried out DLS measurement using the pure reverse micelle solution to study the size distribution profile of the reverse micelle aggregates. Figure 2 shows the size distribution profile of the reverse micelle present in the solution.

DLS result showed that reverse micelle solution has low level of polydispersity with average reverse micelle diameter $\sim 3.9 \mathrm{~nm}$. Then we incorporate the metal precursor, silver nitrate solution inside the reverse micelle keeping the $\mathrm{w}$ value of the solution at $\mathrm{w}=3$ condition. Incorporation of metal precursor sometimes destabilized a reverse micelle solution so I checked the stability of the solution using the same ageing method and it also showed similar stability. To check the impact of metal precursor loading on the reverse micelle size and dispersity profile I again carried out DLS measurement on the metal precursor loaded reverse micelle solution. 


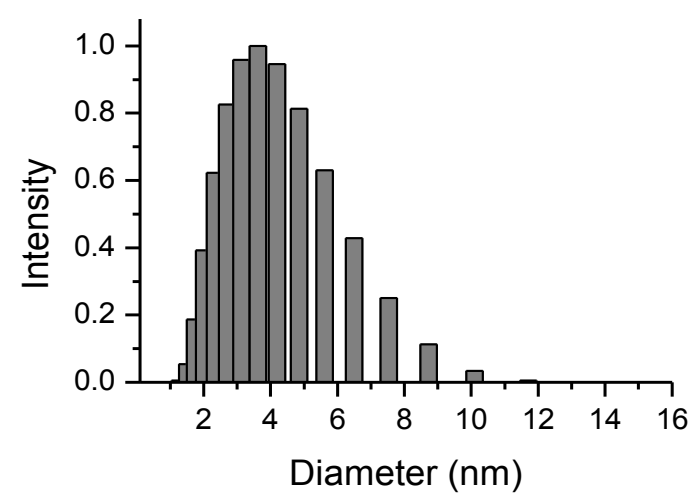

Figure 2. DLS size distribution profile of pure glycerolAOT-n-heptane reverse micelle solution.

Figure 3 shows the DLS size distribution profile of glycerolAOT-n-heptane reverse micelle solution after metal precursor loading where overall concentration of the silver ion in the reverse micelle solution was kept at $4 \times 10^{-3} \mathrm{M}$.

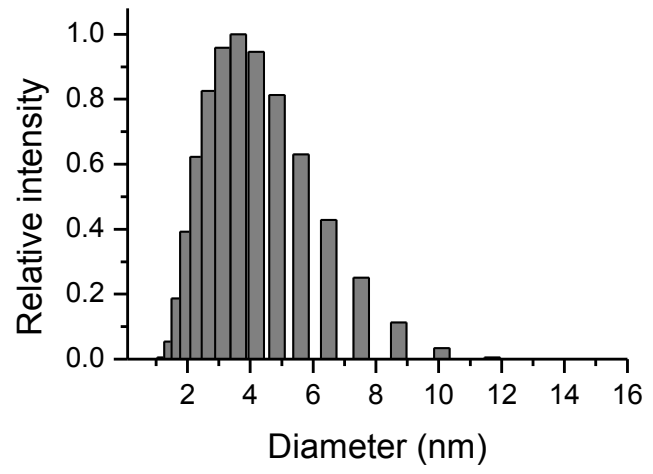

Figure 3. DLS size distribution profile of glycerol-AOT-nheptane reverse micelle solution with overall silver ion concentration $4 \times 10^{-3} \mathrm{M}$.

The result showed that size distribution profile of the reverse micelle solution was almost unchanged with average diameter $\sim 4.0 \mathrm{~nm}$. Then the same solution is used for UVVis spectra recording and TEM analysis. Figure 4 shows the UV-Vis spectrum of the glycerol-AOT-n-heptane reverse micellar solution containing silver nanopartile in the AOT reverse micelle core.

UV-Vis spectrum shows a clear peak $\sim 410 \mathrm{~nm}$ indicating the formation of spherical silver nanoparticle. Figure 5 shows the TEM picture of the silver nanoparticle that was formed inside the glycerol-AOT reverse micelle core. Particle size histogram analysis shows that average particle diameter is $\sim 7 \mathrm{~nm}$.

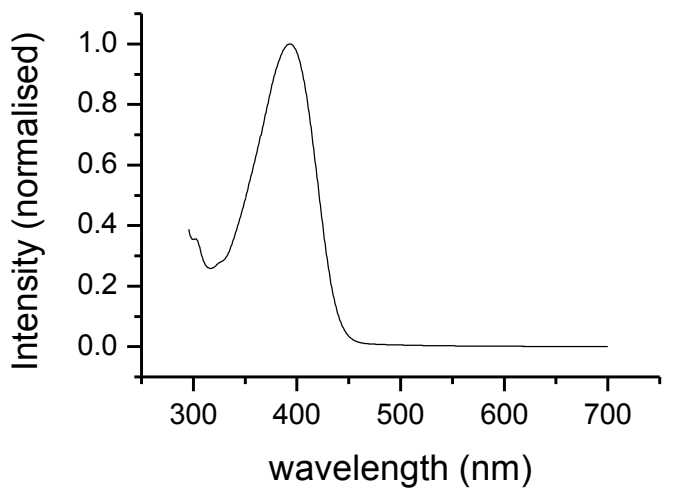

Figure 4. UV-Vis spectrum of glycerol-AOT-n-heptane reverse micellar solution containing silver nanoparticle in the reverse micelle core.

During size analysis I have avoided the large agglomerated structures which are formed due to aggregation of individual small spherical nanoparticle $[11,13]$.

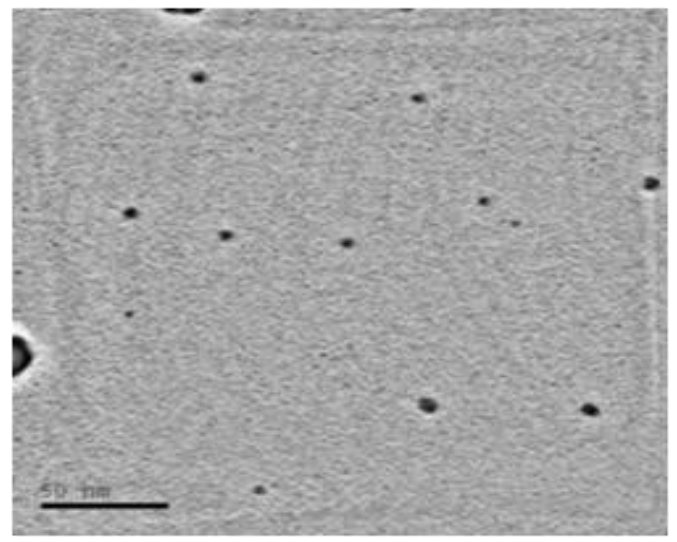

Figure 5. TEM picture of silver nanoparticle synthesized in glycerol AOT n-heptane reverse micelle solution.

\section{DISCUSSION}

DLS results of the pure glycerol-AOT-n-heptane reverse micelle system (figure 2) and silver nitrate precursor loaded reverse micelle system (figure 3) shows that there is no appreciable change in the average reverse micelle size. Average diameter value and size distribution pattern of two profile shows similar distribution with average micelle diameter $\sim 4 \mathrm{~nm}$. This indicates that natural environment of glycerol-AOT reverse micelle is almost unchanged or present in a very less perturbed state. Presence of UV-Vis absorption peak $\sim 410 \mathrm{~nm}$ (Figure 4) indicate the formation of spherical silver nanoparticle in the glycerol AOT reverse micelle system. Absence of absorption shoulder or second peak in the absorption profile also indicates that the formed particle will be of spherical in nature. Spherical shape of reverse micelle aggregate as well as the spherical nature of synthesized silver nanoparticle is a proof that glycerol-AOT reverse micelle is acting as an effective templating media which stabilising the synthesized silver nanoparticle in its 
reverse micelle core. TEM picture (Figure 5) shows the direct evidence of the above statement. Particle size histogram analysis from TEM picture shows an average particle diameter $\sim 7 \mathrm{~nm}$. The value is slightly higher than the pure reverse micelle size. This is due to the perturbation effect exerted by the solid silver nanoparticle present in the reverse micelle core. Such perturbation effect and smaller size difference between pure reverse micelle size and systhesized average particle diameter is also reported other studies $[10,11,13]$.

\section{CONCLUSION AND FUTURE SCOPE}

This study shows that comparatively less explored nonaquesous glycerol-AOT-n-heptane reverse micelle system can be successfully applied as template assisted metal nanoparticle synthesis. Templating effect of AOT-glycerol reverse micelle is strong and it provides sufficient stabilising effect to make the silver nanoparticle solution stable for long time. Slight mismatch of average diameter between nanoparticle and reverse micelle was reported in the previous work [10] on aqueous reverse micelle system. Here, we also observe similar type of diameter mismatch. Average diameter of the pure glycerol reverse micelle was $\sim 4 \mathrm{~nm}$ and the diameter of the synthesised silver nanoparticle was $\sim 7 \mathrm{~nm}$. Accepting this mismatch as common phenomena in reverse micelle nanomaterial synthesis, this nonaqueous reverse micelle system has more scope compared to the water based reverse micelle system because organic reaction and sysnthesis is done in nonaqueous hydrocarbon based solvent.

\section{ACKNOWLEDGMENT}

Author, P. Setua is thankful to Nilmoni Sarkar for his guidance throughout his research life. Author gratefully acknowledge the financial support from UGC minor research project [F.No.PSW-230/15-16 (ERO)], India.

\section{REFERENCES}

[1] A.P. Alivisatos, "Semiconductor clusters, nanocrystals, and quantum dots", Science, Vol. 271, Issue.5251, pp.933-937, 1996.

[2] R.C. Hayward, D.A. Saville, I.A. Aksay, "Electrophoretic assembly of colloidal crystals with optically tunable micropatterns", Nature, Vol. 404, No. 6773, pp.56-59, 2000.

[3] M. Law, D.J. Sirbuly, J.C. Johnson, J. Goldberger, R.J. Saykally, P. Yang, "Nanoribbon waveguides for subwavelength photonics integration", Science, Vol. 305, Issue. 5688, pp. 1269-1273, 2004.

[4] S.A. Maier, M.L. Brongersma, P.G. Kik, S. Meltzer, A.A.G. Requicha, H.A. Atwater, "Plasmonics-A route to nanoscale optical devices" Advanced Materials, Vol. 13, No. 19, pp. 1501$1505,2001$.

[5] [5] R. Narayanan, M.A. El-Sayed, "Catalysis with transition metal nanoparticles in colloidal solution: nanoparticle shape dependence and stability" J. Phy. Chem. B, Vol.109, Issue.26, pp.12663-12676, 2005.

[6] A.K. Salem, P.C. Searson, K.W. Leong, "Multifunctional nanorods for gene delivery" Nat. Mater., Vol.2. No.10, pp.668671, 2003
[7] A.G. Tkachenko, H. Xie, D. Coleman, W. Glomm, J. Rayan, M.F. Anderson, S. Franzen, D.L. Feldheim, "Multifunctional gold nanoparticle-peptide complexes for nuclear targeting”, J. Am. Chem. Soc. Vol.125, Issue.16, pp.4700-4701, 2003.

[8] R. Coontz, P. Szuromi, "Taking the initiative" Vol.290, Issue.5496, pp.1523, 2000.

[9] A. Henglein, "Small-Particle research: Physicochemical properties of extremely small colloidal metal and semiconductor particles", Chem. Rev., Vol.89, Issue.8, pp.1861-1873, 1989.

[10] C. Petit, P. Lixon, M.P. Pileni, "In situ synthesis of silver nanocluster in AOT reverse micelles", J. Phy. Chem., Vol. 97, Issue. 49, pp. 12974-12983.

[11] P. Setua, R. Pramanik, S. Sarkar, C. Ghatak, S.K. Das, N. Sarkar, "Synthesis of silver nanoparticle inside the nonaqueous ethylene glycol reverse micelle and a comparative study to show the effect of the nanoparticle on the reverse micellar aggregates through solvation dynamics and rotational relaxation measurements" $\mathrm{J}$. Phy. Chem. B, Vol. 114, Issue. 22, pp. 7557-7564.

[12] P. Setua, R. Pramanik, S. Sarkar, C. Ghatak, V.G. Rao, N. Sarkar, S.K. Das, "Synthesis of silver nanoparticle in imidazolium and pyrolidium based ionic liquid reverse micelles: A step forward in nanostructure inorganic material in room temperature ionic liquid field", Journal of Molecular Liquids, Vol. 162, Issue. 1, pp. 33-37.

[13] P.Setua, A. Chakraborty, D. Seth, M.U. Bhatta, P.V. Satyam, N. Sarkar, "Synthesis, optical properties, and surface enhanced raman scattering of silver nanoparticle in nonaqueous methanol reversemicelles", J. Phy. Chem. C, Vol. 111, No. 10, pp. 39013907, 2007. and references therein.

[14] M.Y. Khan, M. Roy, "Synthesis, Limitation and Application of gold nanoparticle in treatment of cancerous cell", Int. J. Sci. Res. Multidisciplinary. Studies, Vol. 5, Issue. 9, pp. 08-14, 2019.

\section{AUTHORS PROFILE}

Mr. Palash Setua pursed B. Sc., M.Sc. from Vidyasagar University in 2002 and 2004.He completed his Ph.D. in Physical Science from IIT, Kharagpur in 2011. $\mathrm{He}$ is currently working as Asst. Professor in Department of Chemistry, Pingla Thana Mahavidyalaya, Maligram, Paschim Medinipur, West Bengal since

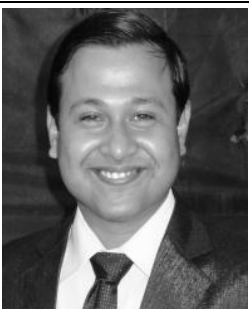
2007. His main research work focuses on steady state and time resolved fluorescence spectroscopy, Reverse micelle and self assembled media and nanomaterial. He has 12 years of teaching experience and 15 years of research experience. 